University of Nebraska - Lincoln

DigitalCommons@University of Nebraska - Lincoln

Faculty Publications from Nebraska Center for Materials and Nanoscience, Nebraska Center Materials and Nanoscience

4-20-2007

\title{
Elasticity of planar fiber networks
}

\author{
X.-F. Wu \\ University of Nebraska - Lincoln, xfwu@unlserve.unl.edu
}

Yuris A. Dzenis

University of Nebraska - Lincoln, ydzenis@unl.edu

Follow this and additional works at: https://digitalcommons.unl.edu/cmrafacpub

Part of the Nanoscience and Nanotechnology Commons

Wu, X.-F. and Dzenis, Yuris A., "Elasticity of planar fiber networks" (2007). Faculty Publications from Nebraska Center for Materials and Nanoscience. 17.

https://digitalcommons.unl.edu/cmrafacpub/17

This Article is brought to you for free and open access by the Materials and Nanoscience, Nebraska Center for (NCMN) at DigitalCommons@University of Nebraska - Lincoln. It has been accepted for inclusion in Faculty Publications from Nebraska Center for Materials and Nanoscience by an authorized administrator of DigitalCommons@University of Nebraska - Lincoln. 


\title{
Elasticity of planar fiber networks
}

\author{
X.-F. Wual and Y. A. Dzenis ${ }^{\text {b) }}$ \\ Department of Engineering Mechanics, Center for Materials Research and Analysis, \\ University of Nebraska-Lincoln, Lincoln, Nebraska 68588-0526
}

(Received 6 December 2004; accepted 26 September 2005; published online 1 November 2005)

\begin{abstract}
A micromechanics model is proposed for the elasticity of planar fiber networks (FNs). The FN is created by random deposition of linearly elastic straight rods within a region. The rods are bonded rigidly at contacts. Under external in-plane loading, the FN deformation consists of fiber bending, elongation, and contraction. An effective constitutive relation for fiber network is developed by averaging the strain energy dissipated by all possible fiber deformations in all directions. Numerical calculations are performed to analyze the effects of fiber aspect ratio and fiber concentration on the effective stiffness of the planar random FN. Finite element analysis (FEA) is performed and compared with the theoretical predictions of the effective FN moduli at several fiber concentrations. FEA results are in good agreement with theoretical predictions. The present model can be used for the prediction of mechanical properties, scaling analysis, and optimization of fiber assemblies. (C) 2005 American Institute of Physics. [DOI: 10.1063/1.2123369]
\end{abstract}

\section{INTRODUCTION}

Due to their high porosity and relatively high mechanical properties, nonwoven fibrous materials have been used in numerous applications and industrial products such as thermal and sound insulators, tissue templates, gas and fluid filters, and various paper products. More recently, nonwoven fibers have been used as electrochemical substrates in battery technologies to provide redundant conductive networks satisfying strict demands of high electrical energy density in low-density battery materials, e.g., Ni/MH (nickel-metal hydride) and Li ion (lithium ion) cells. ${ }^{1,2}$ With the development of nanotechnology, ultrafine continuous fibers produced by electrospinning $^{3-5}$ are also finding promising applications in nanofiber composites, ${ }^{6-8}$ electromagnetic shielding, filtration, and chemical catalyst carriers, ${ }^{9}$ among others. Mechanical properties of these nonwoven fibrous materials are fundamental to their environmental response and intended functions.

A fiber network is a porous structure with fiber volume fraction depending on a specific fiber arrangement. Planar random fiber network $(\mathrm{FN})$ is commonly used to describe many actual fiber assemblies. Such a FN can be modeled by randomly depositing short, straight fiber segments within a domain, as shown in Figs. 1 and 2. Random FNs have been studied by many investigators. Fiber-fiber contact distribution, network permeability, strength, and dynamics have been analyzed. ${ }^{10-15}$ Several models have been dedicated to understanding mechanical properties of fiber assemblies. van $\mathrm{Wyk}^{16}$ derived a power-law pressure-volume relation for the compression of wool with a three-dimensional (3D) random orientation. In this model, the wool assembly was treated as a layered system with fiber bending deflection between neighboring contacts, while no fiber elongation, contraction, or torsion were considered. Based largely on this scheme,

\footnotetext{
${ }^{a)}$ Electronic mail: xfwu@unlserve.unl.edu

${ }^{b)}$ Electronic mail: ydzenis@unl.edu
}

several refined models have been proposed ${ }^{17-26}$ to account for fiber slippage, large deformation, and hybrid fiber systems. The fundamental assumption of these models is the constant force distribution across any FN section, which may conflict with the deformation compatibility of fiber segments in the assembly.

Alternatively, continuum micromechanics (effectivemedium) models based on representative volume element were developed. $\mathrm{Cox}^{27}$ obtained the effective moduli of planar FNs, such as paper. In his model, only the fiber axial deformation was considered. The effective stiffness of the representative area element (RAE) was obtained by averaging the stiffness contributed by fibers in all possible directions within the RAE. Narter et al. ${ }^{28}$ extended Cox's work to $3 \mathrm{D}$ cases. Yet, Cox's model only accounts for the average effect of individual fibers. Neither fiber-fiber bonds nor transverse fiber deflections are involved. As a result, it is applicable to dilute fiber systems. Recently, researchers have begun to consider the effects of fiber contacts and transverse deflection on the mechanical properties of random FNs. Astrom et al. ${ }^{29-31}$ developed an effective constitutive relation for random FNs by differentiating the strain energy dissipated by individual fibers in the RAE with respect to the

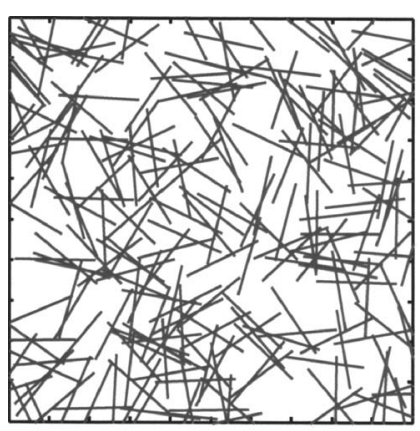

(a)

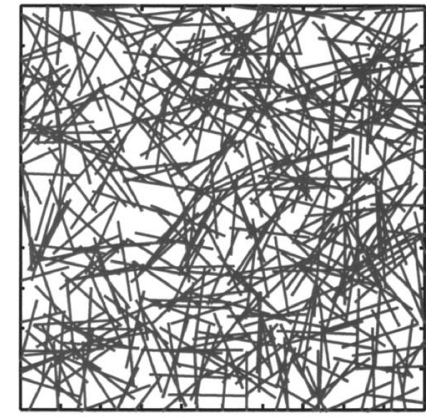

(b)
FIG. 1. Random fiber networks. (a) Fiber concentration: 10 (in fiber length unit); (b) fiber concentration: 20 (in fiber length unit). 


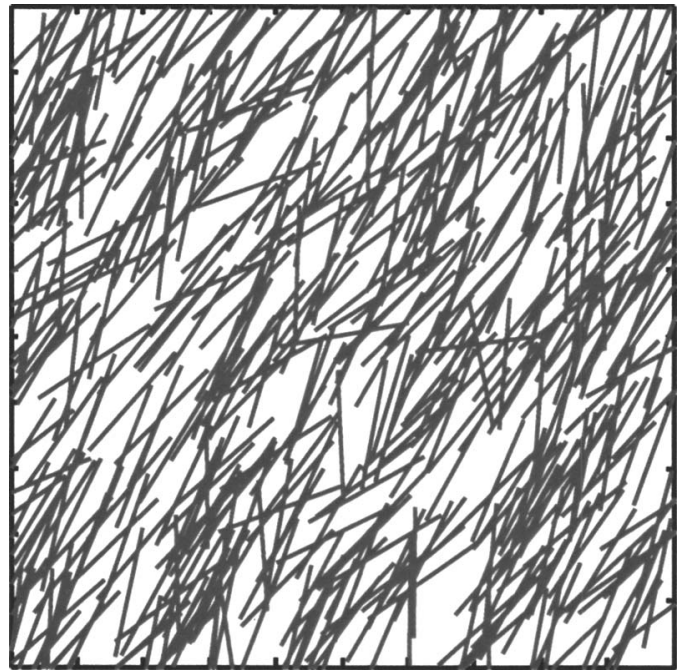

FIG. 2. Fiber network with preferred orientation. (Fiber concentration: 20 (in fiber length unit), mean orientation angle: $60^{\circ}$, and standard deviation: $15^{\circ}$.

applied strain. In this model, fiber segments were considered as elemental Bernoulli-Euler beams or stretching and shearing rods of specified length. The relationship between the applied strains and specific fiber deformations was not given, and the fiber-fiber bond description was not clear. The latter may significantly affect the effective stiffness. Recently, in an attempt to study to stiffness and failure behavior of fiber assemblies for battery applications, Wang et al. ${ }^{1}$ Wang and Sastry, ${ }^{2}$ and Sastry et al. $^{32}$ proposed a double-beam model accounting for fiber bonding. In this model, beam segments can be bonded rigidly or flexibly according to the stiffness of a built-in torsional spring that approximates an actual fiberfiber bond. Two beam models, i.e., Bernoulli-Euler and Timoshenko beams, were used to derive the explicit expressions for the effective moduli. It was shown that the effective moduli were sensitive only to fiber segments of very short lengths. Finite element analysis showed that the above two beam types made no significant difference in the predicted modulus for the fiber aspect ratios higher than 10. Simulation also indicated that the FN deformation was mainly attributed to fiber segments with longer lengths. Using this model, Berhan et al. ${ }^{33,34}$ have recently considered the effective stiffness of nanotube sheets.

The effective stiffness of a planar random FN increases with the increase of its arial density, which can be measured by the number of fibers per unit area (fiber concentration). It is clear that the FN made of short fibers needs a minimum fiber concentration to make it geometrically connected in order to be able to transfer load from fiber to fiber. Numerical simulations ${ }^{31,35-39}$ have indicated that there exists approximately a linear relation between the effective stiffness and the fiber concentration $n$ in the vicinity of the percolation threshold $n_{c}$, around 5.7 (on the basis of unit-square fiber length) for planar random FNs made of uniform fibers.

In this work, we further develop a continuum micromechanics model for determining the general effective stiffness of a planar FN involving transverse fiber deformation. In this model, the fiber-fiber bonds are considered rigid, and the relations between fiber deformations at the microscale and the applied strains are described based on the beam theory. The overall FN deformation complies with the deformation compatibility of the effective continuum. As a result, an explicit relation for the effective stiffness is derived. Effects of the fiber aspect ratio, fiber concentration, and fiber orientation on the effective stiffness are further explored. A finite element analysis (FEA) scheme is developed to examine and validate the present model. Examples are given to show the variation of the effective stiffness and Poisson's ratio of a planar random FN with the structural parameters and fiber concentration.

\section{PROBLEM FORMULATION}

Hereafter we consider a planar FN made of straight fibers of uniform lengths. Figure 1 shows a typical random FN, and Fig. 2 shows a typical FN with a preferred fiber orientation. In an attempt to model its constitutive relation, the planar FN under consideration is idealized as follows. ${ }^{25}$

(1) The FN structure is statistically homogenous.

(2) The fibers are straight and oriented in the same plane (planar FN).

(3) The fibers are well dispersed in space, in the sense that they do not form bundles of parallel fibers.

(4) The fiber diameters and lengths are uniform.

(5) The space between the fibers is considered as void; i.e., there is nothing else there to store or dissipate strain energy.

(6) The deformation is linearly elastic; i.e., no sliding is allowed between fibers at contacts, as this would give rise to inelastic dissipation. This is a necessary condition for the existence of a positive-definite strain energy function, on which the present derivation relies.

(7) All fiber contacts are rigid; i.e., each fiber segment is a beam segment with two ends fixed to other fiber segments, and there is no angular displacement between two segments fixed at the same point.

Since fiber contacts are assumed ideal and no sliding is allowed, each fiber segment between two contacts can be modeled by the Bernoulli-Euler beam. ${ }^{1,2,32}$ For uncorrelated fiber centers, the formation of a random FN made of a large number of fibers with sufficient aspect ratio and fiber concentration may be considered as a planar Poisson process. Therefore, the following probability distribution can be used to describe the fiber segment length distribution, where the segment is a portion of the fiber between two neighboring contacts:

$$
f(l)=\exp (-l / \bar{l}) / \bar{l},
$$

where $\bar{l}$ is both the mean segment length and the standard deviation. Although relation (1) is based on a FN made of fibers with infinite length, it is a good approximation for planar FNs of sufficient density made of fibers with uniform finite lengths. To examine this, we considered the segment length distribution and the relevant density of a random FN with fiber concentration of 20. Computer simulation using Monte Carlo method showed that the simulated segment 


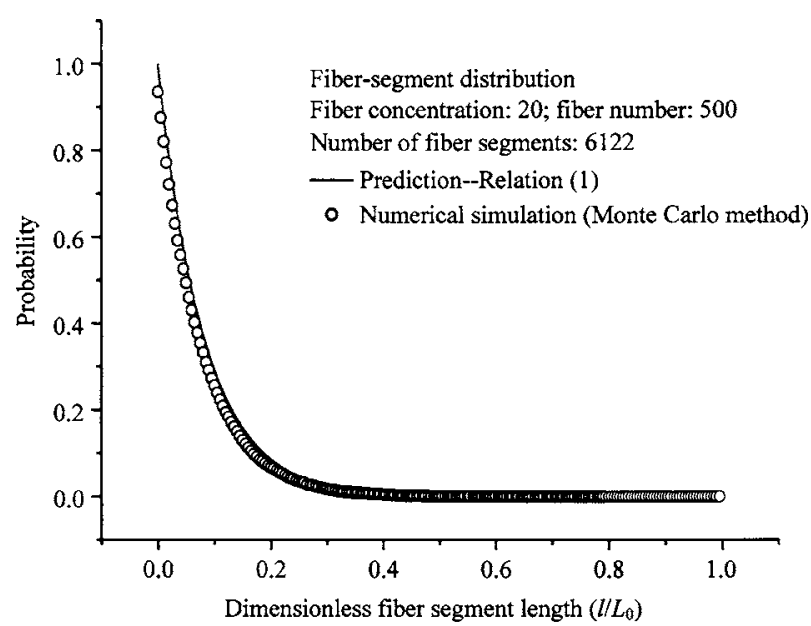

(a)

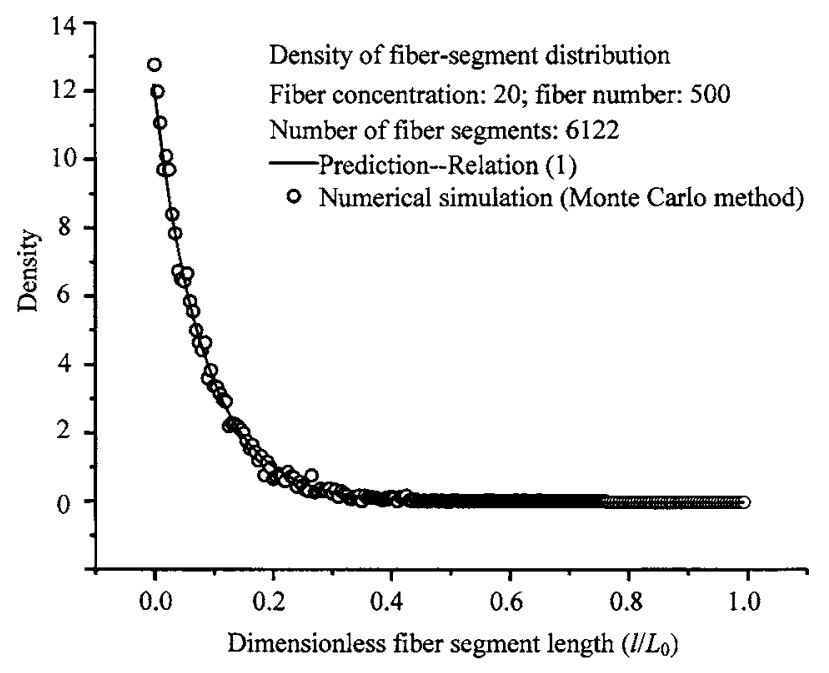

(b)

FIG. 3. Fiber segment length distribution in a random fiber network. (a) Fiber segment length distribution; (b) density of fiber segment length distribution.

length distribution was very close to the one predicted by relation (1), as illustrated in Figs. 3(a) and 3(b). As a result, relation (1) was used for the statistical analysis of the geometrical and physical properties of random fiber networks.

Let us first consider a general planar FN characterized by fiber concentration $n$ and fiber angular and length distribution $\psi(\theta, L)$. Here $\theta$ is the fiber orientational angle (an angle between the fiber axis and the horizontal line) and $L$ is the fiber length. The fiber radius $r$ is considered uniform and the fiber concentration $n$ is defined as the number of fibers per unit area. $\theta$ and $L$ are assumed uncorrelated, thus $\psi(\theta, L)$ can be written as

$$
\psi(\theta, L)=\varphi(\theta) g(L)
$$

where $\varphi(\theta)$ and $g(L)$ are the fiber angular and length distribution functions, respectively. Specifically, for a FN made of fibers with uniform lengths $L_{0}$ and uniformly distributed in the interval $\left[0, \theta_{0}\right], \psi(\theta, L)$ is in the form

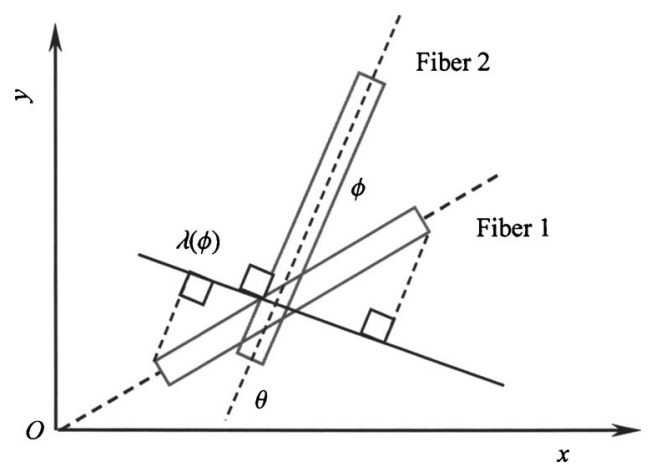

FIG. 4. Schematic fiber contact analysis by the tube model.

$$
\psi(\theta, L)=\delta\left(L-L_{0}\right) / \theta_{0}, \quad\left(0<\theta_{0}<\pi\right)
$$

where $\delta\left(L-L_{0}\right)$ is the Dirac delta function and $L_{0}$ is the fiber length.

Here we estimate the average number of contacts per fiber by using a tube model. ${ }^{40}$ For a planar FN made of straight fibers, the projection of each fiber on the network plane is a rectangle of area $L \times 2 r$. The concentration of fibers with length $L$ and orientational angle $\theta$ is $n \psi(\theta, L)$. Therefore, fibers intersecting a fixed fiber have their midpoints within an area $\lambda(\phi) L+A_{0}$, where $\phi$ is the angle between the fixed fiber and the one with orientation angle $\theta$, as shown in Fig. 4. The area $A_{0}=2 r L_{0}$ is the projection of the fixed fiber in the FN plane, and $\lambda(\phi)$ is the projected length of the fixed fiber on a straight line perpendicular to the fiber with orientation $\theta$. Consequently, the number of intersecting fibers with lengths in the range of $[L, L+d L]$ and orientation in the range of $[\phi, \phi+d \phi]$ can be estimated as

$$
d N(\phi, L)=n L_{0} \psi(\phi, L)[\lambda(\phi)+2 r] d L d \phi
$$

Integrating relation (4) over all possible fiber lengths (uniform $L_{0}$ in the present case) and orientations yields the average contact number per fiber:

$$
N=n L_{0}[\langle\lambda\rangle+2 r]
$$

with

$$
\langle\lambda\rangle=\int_{0}^{\theta_{0}} \int_{0}^{\infty} \psi(\phi, L) \lambda(\phi) d L d \phi .
$$

In the present case of uniform fiber lengths $L_{0}, \lambda(\phi)$ can be expressed as

$$
\lambda(\phi)=L_{0} \sin (\phi)+2 r|\cos (\phi)|
$$

where $|\cdot|$ denotes the absolute value. For distribution (3), the mean number of contacts per fiber can be estimated from (4)-(7) as 


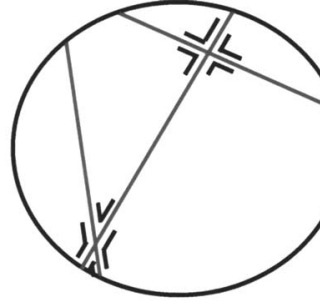

(a)
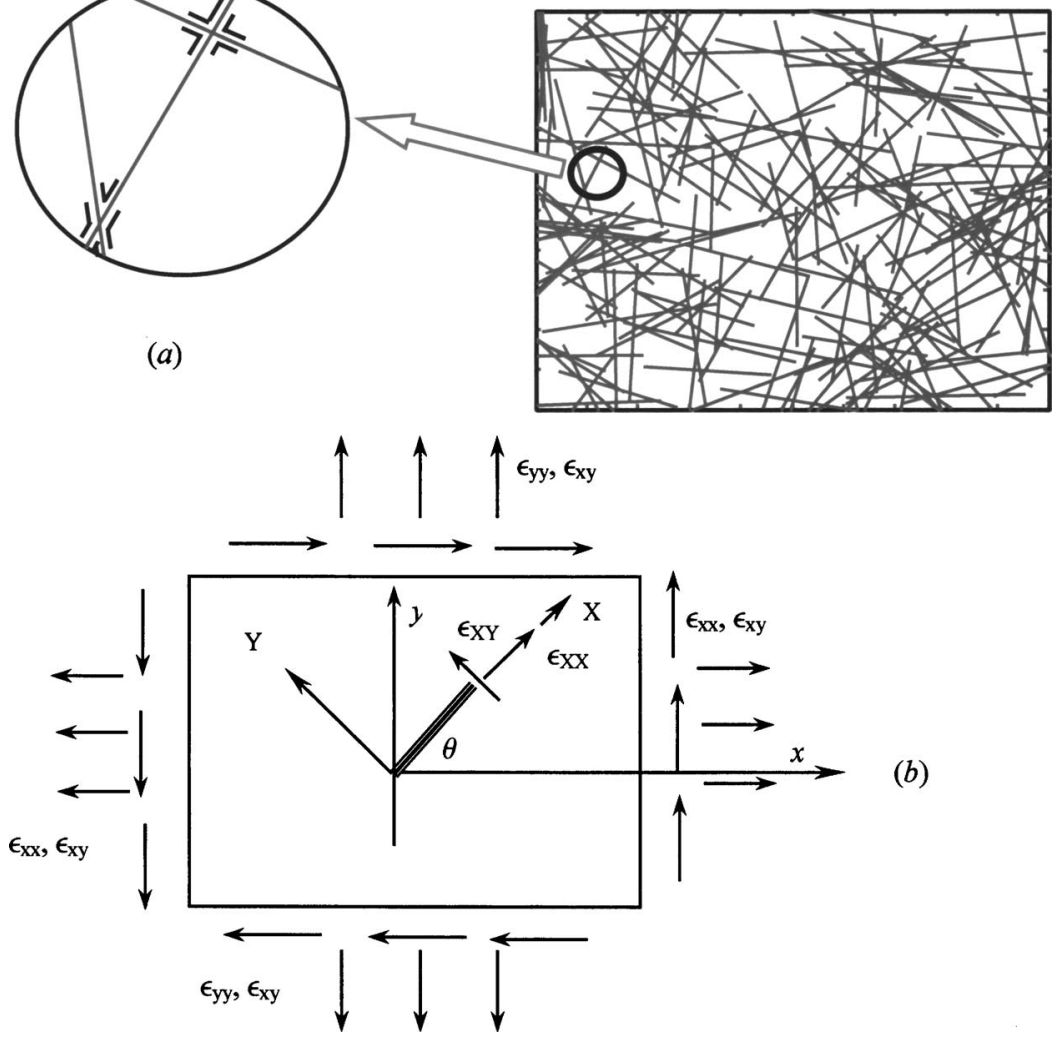

(b)

FIG. 5. Representative region of a planar fiber network.

$$
N=\left\{\begin{array}{cl}
n L_{0}^{2}\left\{1-\cos \left(\theta_{0}\right)+2\left[\theta_{0}+\sin \left(\theta_{0}\right)\right] r / L_{0}\right\} / \theta_{0}, & 0<\theta_{0} \leqslant \pi / 2, \\
n L_{0}^{2}\left\{1-\cos \left(\theta_{0}\right)+2\left[2+\theta_{0}-\sin \left(\theta_{0}\right)\right] r / L_{0}\right\} / \theta_{0}, & \pi / 2 \leqslant \theta_{0} \leqslant \pi .
\end{array}\right.
$$

For fibers uniformly distributed in the entire plane, i.e., $\theta_{0}$ $=\pi$, the above relation reduces to

$$
N=2 n L_{0}^{2}\left[1+(2+\pi) r / L_{0}\right] / \pi
$$

To examine relation (9), we performed the Monte Carlo simulation of random FNs at several fiber concentrations with $r / L_{0} \rightarrow 0$. For $n L_{0}^{2}>10$, numerical results showed that the deviation was within $5 \%$ for a testing region with fiber number above 500. With the number of average contacts $N$ per fiber, the mean segment length can be estimated as

$$
\bar{l}=L_{0} /(N+1) .
$$

Substituting (9) into (10) leads to

$$
\bar{l}=L_{0} /\left\{1+2\left(n L_{0}^{2}\right)\left[1 / \pi+(1+2 / \pi) r / L_{0}\right]\right\} .
$$

In an attempt to study the effective moduli of planar FNs, the RAE concept commonly used in micromechanics ${ }^{41,42}$ may be adopted. In this concept the RAE is a statistical representative area of the planar FN. In this work, we consider the effective FN deformations based on the elastic deformation of a single fiber segment statisti- cally. Accordingly, we assume that the microscopic deformations of a fiber segment comply with the strain compatibility of the effective continuum medium. This is necessary to derive a relation bridging the fiber segment deformations with the macroscopic strains in the effective medium. The macroscopic stresses and strains in the effective continuum medium are assumed to be uniform. The effective moduli can be then determined based on the equivalency of the strain energy dissipated by the fibers oriented in all possible directions in a given region to that of the effective continuum medium. Without loss of generality, we consider a rectangular region $L_{x} \times L_{y}$ of the effective continuum medium, which is assumed anisotropic. The generalized stress-strain relation is described as follows:

$$
\left[\begin{array}{c}
\sigma_{x x} \\
\sigma_{y y} \\
\tau_{x y}
\end{array}\right]=\left[\begin{array}{lll}
Q_{11} & Q_{12} & Q_{13} \\
Q_{12} & Q_{22} & Q_{23} \\
Q_{13} & Q_{23} & Q_{33}
\end{array}\right]\left[\begin{array}{c}
\varepsilon_{x x} \\
\varepsilon_{y y} \\
\gamma_{x y}
\end{array}\right]
$$

For a given planar strain $\left(\varepsilon_{x x}, \varepsilon_{y y}, \varepsilon_{x y}\right)$ as shown in Fig. 5, the strain energy dissipated within the given region can be calculated as 


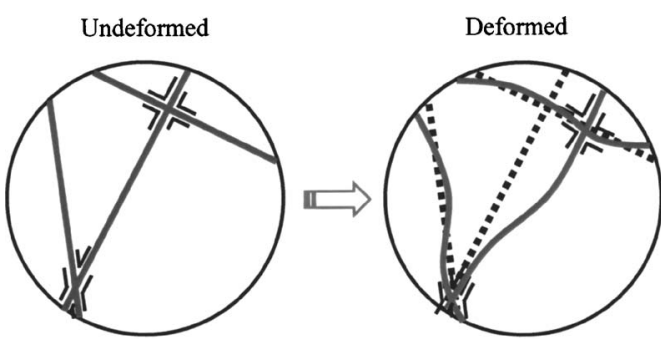

(a)

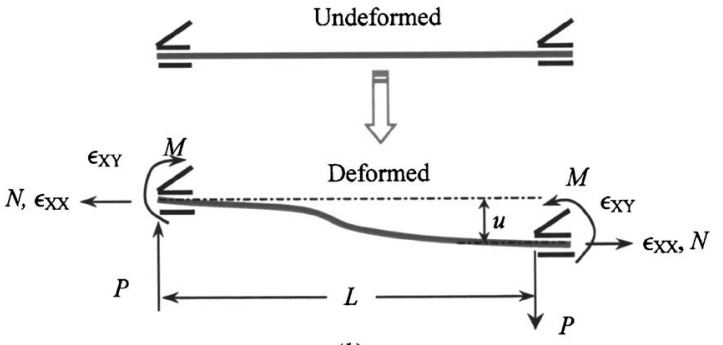

(b)

$$
\begin{aligned}
U= & \frac{1}{2}\left[\begin{array}{c}
\sigma_{x x} \\
\sigma_{y y} \\
\tau_{x y}
\end{array}\right] T\left[\begin{array}{c}
\varepsilon_{x x} \\
\varepsilon_{y y} \\
\gamma_{x y}
\end{array}\right] L_{x} L_{y} t=\frac{1}{2}\left[\begin{array}{c}
\varepsilon_{x x} \\
\varepsilon_{y y} \\
\gamma_{x y}
\end{array}\right] T\left[\begin{array}{lll}
Q_{11} & Q_{12} & Q_{13} \\
Q_{12} & Q_{22} & Q_{23} \\
Q_{13} & Q_{23} & Q_{33}
\end{array}\right] \\
& \times\left[\begin{array}{c}
\varepsilon_{x x} \\
\varepsilon_{y y} \\
\gamma_{x y}
\end{array}\right] L_{x} L_{y} t
\end{aligned}
$$

where $L_{x}$ and $L_{y}$ are the length and width of the rectangular region, which may be set as unit lengths for convenience, and $t$ is the thickness. Here $t=2 r$ for planar FN made of uniform fibers of radius $r$. Consider a typical fiber segment with arbitrary orientation angle $\theta$ (Fig. 5). Let us introduce a local coordinate system $(X, Y)$ with $X$ directed along the fiber axis and $Y$ perpendicular to the fiber axis. The corresponding local axial and shear strains are ${ }^{43}$

$$
\begin{aligned}
& \varepsilon_{X X}=\frac{\varepsilon_{x x}+\varepsilon_{y y}}{2}+\frac{\varepsilon_{x x}-\varepsilon_{y y}}{2} \cos (2 \theta)+\varepsilon_{x y} \sin (2 \theta), \\
& \varepsilon_{X Y}=-\frac{\varepsilon_{x x}-\varepsilon_{y y}}{2} \sin (2 \theta)+\varepsilon_{x y} \cos (2 \theta) .
\end{aligned}
$$

Microscopically, under external strain, the fiber segments within the FN will be deformed in several ways. In general, the fiber segments will be deformed in a way that offers the least elastic resistance, i.e., stores the least strain energy via coupled bending, shearing, stretching, and twisting. For a circular fiber segment, its bending stiffness is $3 \pi E r^{4} / l^{3}$, its shear stiffness $\pi E r^{2} /,[2(1+\nu) l]$, and its elongation modulus $\pi E r^{2} / l$. In this study, coupled deformations are considered for an individual fiber segment, similar to the ones considered in previous studies. ${ }^{12,13}$ Specifically, it is assumed that a fiber segment deforms by coupled bending, shearing, and stretching if the fiber bending stiffness is lower than both the shear and elongation moduli, i.e., if the fiber segment length satisfies $l>l_{C}=r \sqrt{6(1+\nu)}$. In the case of $l<l_{C}$, a fiber segment deforms by only shearing and stretching.

Therefore, for a long fiber segment with $l>l_{C}$, the effective shear strain $\varepsilon_{X Y}$ is approximated as
FIG. 6. Deformations of a single fiber segment. [(a) and (b)] Bending, shearing, and stretching; (c) stretching and shearing.

$$
\varepsilon_{X Y}=\gamma_{X Y} / 2=u /(2 l),
$$

where $u$ is the fiber deflection as shown in Fig. 6. Under the assumption of rigid contacts, the strain energy dissipated by the fiber segment bending, shearing, and stretching can be evaluated as

$$
U_{L}=3 \pi E r^{4}\left(\gamma_{X Y}\right)^{2} /(2 l)+\pi E r^{2} l\left(\varepsilon_{X X}\right)^{2} / 2,
$$

where the fiber material is assumed to be isotropic and linearly elastic and $E$ is the fiber Young modulus. For a short fiber segment with $l<l_{C}$, the strain energy dissipated by shearing and stretching is calculated as

$$
U_{S}=\pi E r^{2} l\left(\varepsilon_{X X}\right)^{2} / 2+\pi E r^{2} l\left(\gamma_{X Y}\right)^{2} /[4(1+\nu)],
$$

where $\nu$ is the Poisson ratio of the fiber segment and $\varepsilon_{X X}$ and $\varepsilon_{X Y}$ are the local effective strains as shown in Fig. 5. The number of fibers in the rectangular region under consideration is

$$
N_{0}=n L_{x} L_{y}
$$

thus the number of total fiber segments in this region can be determined according to (5) as

$$
N_{s}=n\left\{n L_{0}[\langle\lambda\rangle+2 r]-1\right\} L_{x} L_{y}
$$

where the dangled segments at fiber ends have been excluded. With the aid of relation (1), the strain energy dissipated by all fiber segments in the given region is

$$
\begin{aligned}
U= & n \pi E r^{2} L_{x} L_{y} L_{0} \int_{0}^{\theta_{0}} \frac{\left(\varepsilon_{X X}\right)^{2}}{2} \varphi(\theta) d \theta \\
& +n \pi E\left\{n L_{0}[\langle\lambda\rangle+2 r]-1\right\} r^{2} L_{x} L_{y} \\
& \times\left\{\int_{0}^{\theta_{0}} \int_{0}^{l_{C}} \frac{\left(\gamma_{X Y}\right)^{2}}{4(1+\nu)} \psi(\theta, L) f(L) L d L d \theta\right. \\
& \left.+3 r^{2} / 2 \int_{0}^{\theta_{0}} \int_{l_{C}}^{L_{0}} \frac{\left(\gamma_{X Y}\right)^{2}}{L} \psi(\theta, L) f(L) d L d \theta\right\},
\end{aligned}
$$

where the first term is stretching energy, independent of fiber contacts, i.e., Cox's term, and the second and third terms are 
relevant to the shearing and bending energies. In the case of a planar FN made of fibers with uniform lengths and distributed uniformly in the interval $\left[0, \theta_{0}\right]$, substitution of (3) into (20) leads to

$$
\begin{aligned}
U= & n \pi E r^{2} L_{x} L_{y} L_{0} \int_{0}^{\theta_{0}} \frac{\left(\varepsilon_{X X}\right)^{2}}{2} \varphi(\theta) d \theta \\
& +n \pi E\left\{n^{2} L_{0}^{2}[\langle\lambda\rangle+2 r]^{2}-1\right\} r^{2} L_{0} L_{x} L_{y} \\
& \times\left\{\frac{1}{N^{2}} \int_{0}^{\theta_{0}} \int_{0}^{N\left(l_{C} L_{0}\right)} \frac{\left(\gamma_{X Y}\right)^{2}}{4(1+\nu)} \eta \exp (-\eta) \varphi(\theta) d \eta d \theta\right. \\
& +\frac{3 r^{2}}{2 L_{0}^{2}} \int_{0}^{\theta_{0}} \int_{N\left(l_{C} / L_{0}\right)}^{N} \frac{\left(\gamma_{X Y}\right)^{2}}{2 \eta} \\
& \times \exp (-\eta) \varphi(\theta) d \eta d \theta\} .
\end{aligned}
$$

Consequently, the constitutive relations for the planar FN can be determined from (13):

$$
\begin{aligned}
\sigma_{x x} & =\frac{1}{2 r L_{x} L_{y}} \frac{\partial U}{\partial \varepsilon_{x x}}, \quad \sigma_{y y}=\frac{1}{2 r L_{x} L_{y}} \frac{\partial U}{\partial \varepsilon_{y y}}, \\
\tau_{x y} & =\frac{1}{2 r L_{x} L_{y}} \frac{\partial U}{\partial \gamma_{x y}} .
\end{aligned}
$$

As an example, we derive the following relations based on the strain energy in the special form (21). Substitution of (21) into (22) yields the effective constitutive relations:

$$
\begin{aligned}
\sigma_{x x}= & n \pi E r L_{0} \int_{0}^{\theta_{0}} \frac{\varepsilon_{X X}}{2} \frac{\partial \varepsilon_{X X}}{\partial \varepsilon_{x x}} \varphi(\theta) d \theta \\
& +n \pi E\left\{n^{2} L_{0}^{2}[\langle\lambda\rangle+2 r]^{2}-1\right\} r L_{0}\left(\frac{\alpha}{1+\nu}+\beta\right) \\
& \times \int_{0}^{\theta_{0}} \gamma_{X Y} \frac{\partial \gamma_{X Y}}{\partial \varepsilon_{x x}} \varphi(\theta) d \theta, \\
\sigma_{y y}= & n \pi E r L_{0} \int_{0}^{\theta_{0}} \frac{\varepsilon_{X X}}{2} \frac{\partial \varepsilon_{X X}}{\partial \varepsilon_{y y}} d \theta \\
& +n \pi E\left\{n^{2} L_{0}^{2}[\langle\lambda\rangle+2 r]^{2}-1\right\} r L_{0}\left(\frac{\alpha}{1+\nu}+\beta\right) \\
& \times \int_{0}^{\theta_{0}} \gamma_{X Y} \frac{\partial \gamma_{X Y}}{\partial \varepsilon_{y y}} \varphi(\theta) d \theta, \\
& +n \pi E\left\{n^{2} L_{0}^{2}[\langle\lambda\rangle+2 r]^{2}-1\right\} r L_{0}\left(\frac{\alpha}{1+\nu}+\beta\right) \\
\tau_{x y}= & n \pi E r L_{0} \int_{0}^{\theta_{0}} \frac{\varepsilon_{X X}}{2} \frac{\partial \varepsilon_{X X}}{\partial \gamma_{x y}} d \theta \\
& \times \int_{0}^{\theta_{0}} \frac{\partial \gamma_{X Y}}{\partial \gamma_{x y}} d \theta . \\
& \\
& \\
&
\end{aligned}
$$

In the above expressions, the partial derivatives can be written as

$$
\begin{aligned}
& \partial \varepsilon_{X X} / \partial \varepsilon_{x x}=\cos ^{2}(\theta), \quad \partial \gamma_{X Y} / \partial \varepsilon_{x x}=-2 \sin (\theta) \cos (\theta), \\
& \partial \varepsilon_{X X} / \partial \varepsilon_{y y}=\sin ^{2}(\theta), \quad \partial \gamma_{X Y} / \partial \varepsilon_{y y}=2 \sin (\theta) \cos (\theta), \\
& \partial \varepsilon_{X X} / \partial \gamma_{x y}=\sin (2 \theta) / 2, \quad \partial \gamma_{X Y} / \partial \gamma_{x y}=\cos (2 \theta),
\end{aligned}
$$

where $\alpha$ and $\beta$ are two dimensionless numbers defined as follows:

$$
\begin{aligned}
\alpha & =\frac{1}{4(N+1)^{2}} \int_{0}^{(N+1)\left(l_{C} / L_{0}\right)} \eta \exp (-\eta) d \eta \\
& =\frac{1-\left[1+(N+1) l_{C} / L_{0}\right] \exp \left[-(N+1) l_{C} / L_{0}\right]}{4(N+1)^{2}}, \\
\beta & =\frac{3 r^{2}}{2 L_{0}^{2}} \int_{(N+1)\left(l_{C} / L_{0}\right)}^{N+1} \frac{\exp (-\eta)}{\eta} d \eta .
\end{aligned}
$$

With the aid of (12), the stiffness components can be determined explicitly through integrating (24) and (25):

$$
\begin{aligned}
& Q_{11}=\frac{n \pi E r L_{0}}{8 \theta_{0}}\left[\frac{3}{2} \theta_{0}+\sin \left(2 \theta_{0}\right)+\frac{\sin \left(4 \theta_{0}\right)}{8}\right] \\
& +\frac{n \pi E\left\{n^{2} L_{0}^{2}[\langle\lambda\rangle+2 r]^{2}-1\right\} r L_{0}}{2 \theta_{0}}\left(\frac{\alpha}{1+\nu}+\beta\right) \\
& \times\left[\theta_{0}-\frac{\sin \left(4 \theta_{0}\right)}{4}\right] \text {, } \\
& Q_{12}=\frac{n \pi E r L_{0}}{16 \theta_{0}}\left[\theta_{0}-\frac{\sin \left(4 \theta_{0}\right)}{4}\right] \\
& -\frac{n \pi E\left\{n^{2} L_{0}^{2}[\langle\lambda\rangle+2 r]^{2}-1\right\} r L_{0}}{2 \theta_{0}}\left(\frac{\alpha}{1+\nu}+\beta\right) \\
& \times\left[\theta_{0}-\frac{\sin \left(4 \theta_{0}\right)}{4}\right] \text {, } \\
& Q_{13}=\frac{n \pi E r L_{0}}{16 \theta_{0}}\left[3+\cos \left(2 \theta_{0}\right)\right] \sin ^{2}\left(\theta_{0}\right) \\
& -\frac{n \pi E\left\{n^{2} L_{0}^{2}[\langle\lambda\rangle+2 r]^{2}-1\right\} r L_{0}}{2 \theta_{0}}\left(\frac{\alpha}{1+\nu}+\beta\right) \\
& \times\left[1+\cos \left(2 \theta_{0}\right)\right] \sin ^{2}\left(\theta_{0}\right), \\
& Q_{22}=\frac{n \pi E r L_{0}}{8 \theta_{0}}\left[\frac{3}{2} \theta_{0}-\sin \left(2 \theta_{0}\right)+\frac{\sin \left(4 \theta_{0}\right)}{8}\right] \\
& +\frac{n \pi E\left\{n^{2} L_{0}^{2}[\langle\lambda\rangle+2 r]^{2}-1\right\} r L_{0}}{2 \theta_{0}}\left(\frac{\alpha}{1+\nu}+\beta\right) \\
& \times\left[\theta_{0}-\frac{\sin \left(4 \theta_{0}\right)}{4}\right] \text {, }
\end{aligned}
$$




$$
\begin{aligned}
Q_{23}= & \frac{n \pi E r L_{0}}{16 \theta_{0}}\left[1-\cos \left(2 \theta_{0}\right)\right] \sin ^{2}\left(\theta_{0}\right) \\
& +\frac{n \pi E\left\{n^{2} L_{0}^{2}[\langle\lambda\rangle+2 r]^{2}-1\right\} r L_{0}}{2 \theta_{0}}\left(\frac{\alpha}{1+\nu}+\beta\right) \\
& \times\left[1+\cos \left(2 \theta_{0}\right)\right] \sin ^{2}\left(\theta_{0}\right), \\
Q_{33}= & \frac{n \pi E r L_{0}}{16 \theta_{0}}\left[\theta_{0}-\frac{\sin \left(4 \theta_{0}\right)}{4}\right] \\
& +\frac{n \pi E\left\{n^{2} L_{0}^{2}[\langle\lambda\rangle+2 r]^{2}-1\right\} r L_{0}}{2 \theta_{0}}\left(\frac{\alpha}{1+\nu}+\beta\right) \\
& \times\left[\theta_{0}+\frac{\sin \left(4 \theta_{0}\right)}{4}\right] .
\end{aligned}
$$

Clearly, the effective elastic behavior of a planar FN made of uniform fibers randomly distributed in the interval $\left[0, \theta_{0}\right]$ is anisotropic. The effective stiffness of the fibrous medium $[(28)-(33)]$ is closely linked to the parameters $n, r, L_{0}$, and $\theta_{0}$.

As an example, we further consider the case of random planar FN, i.e., $\theta=\pi$. In this case, the effective continuum medium is isotropic, and the nontrivial components in the stiffness matrix are

$$
\begin{aligned}
Q_{11} / E=Q_{22} / E= & \pi\left(n L_{0}^{2}\right) / \rho \\
& \times\left\{\frac{3}{16}+2\left[\left(n L_{0}^{2}\right)^{2}\left(\frac{1}{\pi}+\frac{1+2 / \pi}{\rho}\right)^{2}-\frac{1}{4}\right]\right. \\
& \left.\times\left(\frac{\alpha}{1+\nu}+\beta\right)\right\}, \\
Q_{12} / E= & \pi\left(n L_{0}^{2}\right) / \rho\left\{\frac{1}{16}-2\left[\left(n L_{0}^{2}\right)^{2}\left(\frac{1}{\pi}+\frac{1+2 / \pi}{\rho}\right)^{2}-\frac{1}{4}\right]\right. \\
& \left.\times\left(\frac{\alpha}{1+\nu}+\beta\right)\right\}, \\
Q_{33} / E= & \pi\left(n L_{0}^{2}\right) / \rho\left\{\frac{1}{16}+2\left[\left(n L_{0}^{2}\right)^{2}\left(\frac{1}{\pi}+\frac{1+2 / \pi}{\rho}\right)^{2}-\frac{1}{4}\right]\right. \\
& \left.\times\left(\frac{\alpha}{1+\nu}+\beta\right)\right\},
\end{aligned}
$$

where $\rho=L_{0} / r$ is the fiber aspect ratio. The effective Young modulus and Poisson ratio can be extracted from (34)-(36):

$$
\begin{aligned}
& E_{e} / E=\pi\left(1-\nu_{e}^{2}\right)\left(n L_{0}^{2}\right) / \rho\left\{\frac{3}{16}+2\left[\left(n L_{0}^{2}\right)^{2}\left(\frac{1}{\pi}+\frac{1+2 / \pi}{\rho}\right)^{2}-\frac{1}{4}\right]\left(\frac{\alpha}{1+\nu}+\beta\right)\right\}, \\
& \nu_{e}=\frac{\pi^{2}-32\left\{\left(n L_{0}^{2}\right)^{2}[1 / \pi+(1+2 / \pi) / \rho]-1 / 4\right\}[\alpha /(1+\nu)+\beta]}{3 \pi^{2}+32\left\{\left(n L_{0}^{2}\right)^{2}[1 / \pi+(1+2 / \pi) / \rho]-1 / 4\right\}[\alpha /(1+\nu)+\beta]} .
\end{aligned}
$$

For a dilute random FN, (37) and (38) reduce to

$$
E_{e} / E=\pi\left(n L_{0}^{2}\right) /(6 \rho), \quad \nu_{e}=1 / 3,
$$

which coincides with the results of Cox's model. ${ }^{27}$

In the above derivation all fibers were considered connected and deformed, and two dangled segments at the ends of each fiber were excluded as they are free of deformations. Thus, this model can be used for predicting the effective moduli of random FNs with sufficient fiber concentration. At low fiber concentration, most fibers are not connected. When the fiber concentration is below percolation threshed $n_{c}$, the effective moduli are zero. For fiber concentrations close to $n_{c}$, Astrom et al. ${ }^{29-31}$ introduced an ad hoc shifting function for the effective FN moduli. However, at high fiber concentrations, the effective moduli are independent of the percolation threshold $n_{c}$ since all fibers are connected. We will further discuss this issue, analyzing FEA results, in Sec. III.

Finally, we would like to mention that the dimensionless numbers $\alpha$ and $\beta$ in (27) are only functions of fiber aspect ratio $\rho$ and fiber concentration $n L_{0}^{2}$. Therefore, from relations (28)-(39), it can be concluded that there exists a scaling law for the effective stiffness of the planar FNs; i.e., the dimen- sionless effective stiffness $Q_{i j} / E$ and the effective Poisson ratio $\nu_{e}$ are only functions of the dimensionless fiber concentration $n L_{0}^{2}$, fiber aspect ratio $\rho$, and fiber Poisson ratio $\nu$.

\section{NUMERICAL EXAMPLES OF ELASTIC SCALING AND COMPARISON WITH FEA SIMULATIONS}

Let us first consider the variation of the dimensionless effective Young modulus $E_{e} / E$ and Poisson ratio $\nu_{e}$ of a quasiisotropic FN described by (37) and (38) with respect to fiber concentration $n L_{0}^{2}$ at several fiber aspect ratios $\rho$. The Poisson ratio of an individual fiber is selected as 0.33 , thus the length threshold of fiber segments is $l_{C}=r \sqrt{6(1+\nu)}$ $\approx 2.8285 r$. The mean number of fiber contacts $N$ according to (9) is $N=2 n L_{0}^{2}[1+(2+\pi) / \rho] / \pi$. For planar FN, the fiber projected area fraction is approximated as $2 n r / L_{0}$, therefore numerical simulations can be used to approach a realistic FN only for $n r L_{0}<0.5$. Figures 7 and 8 show the corresponding results. It can be observed that the effective modulus increases with the increase of $n L_{0}^{2}$ at fixed fiber aspect ratios, while it decreases steeply with the decrease of fiber aspect ratio $\rho$ at a given $n L_{0}^{2}$. Since the current model involves transverse fiber deformation, the effective modulus is higher 


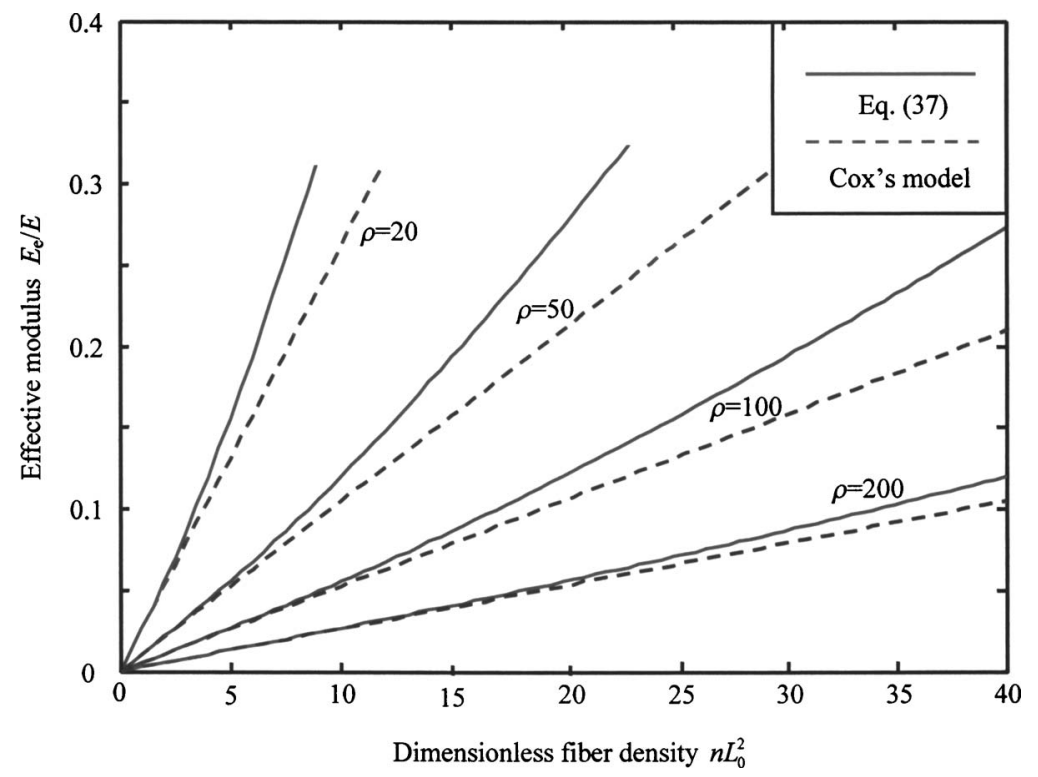

FIG. 7. Variation of dimensionless effective modulus $E_{e} / E$ with fiber concentration $n L_{0}^{2}$ and fiber aspect ratio $\rho$.

than the one predicted by Cox's model. The effective Poisson ratio $\nu_{e}$ decreases with the increase of $n L_{0}^{2}$ at fixed fiber aspect ratios, while it also increases with the increase of fiber aspect ratio at a given $n L_{0}^{2}$. When $n L_{0}^{2}$ nears the percolation threshold $n_{c} L_{0}^{2}$, many fibers are not connected. In this case, relation (37) is no longer valid.

To examine the above model, we performed a finite element analysis of a random FN at several fiber concentrations. The FN fiber aspect ratio was fixed at $L_{0} / r=100$. During the simulation, each random FN was created within a square region with sides five times the length of the single fiber. Symmetrical displacement boundary conditions were used, and a beam element with transverse shear was selected. All fiber segments were considered rigidly bonded at contacts. In the FEA model, any two nodes located at a distance lower than the fiber radius were merged in order to eliminate numerical difficulties. FEA results are plotted in Fig. 9 Based on relation (37), a modified formula for the effective modulus was used:

$$
\begin{aligned}
E_{e} / E= & {\left[1-\exp \left(\frac{1-n / n_{c}}{2}\right)\right] \pi\left(1-\nu_{e}^{2}\right)\left(\frac{n L_{0}^{2}}{\rho}\right) } \\
& \times\left\{\frac{3}{16}+2\left[\left(n L_{0}^{2}\right)^{2}\left(\frac{1}{\pi}+\frac{1+2 / \pi}{\rho}\right)^{2}-\frac{1}{4}\right]\right. \\
& \left.\times\left(\frac{\alpha}{1+\nu}+\beta\right)\right\} .
\end{aligned}
$$

In this relation, an ad hoc exponential decay function is introduced to approximate the numerical data close to the percolation threshold $n_{c}$, and the parameter $l_{c}$ is selected as $0.01 r$, which is reasonable for the current simulation since all fiber segments are considered as beam elements in the FEA. Figure 9 shows that the numerical results based on FEA are close to the theoretical predictions. Therefore, the present model gives a good approximation of the effective stiffness of planar random FNs. At low fiber concentrations, the shifting function can be used. Note that at high fiber concentra-

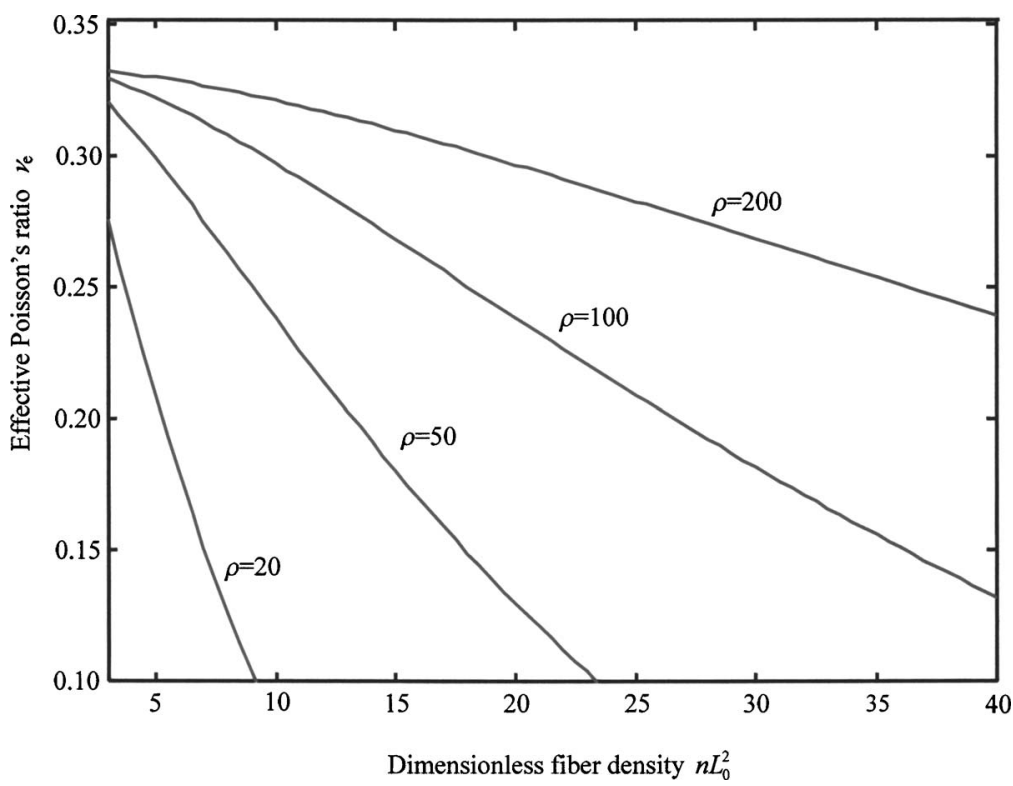

FIG. 8. Variation of effective Poisson's ratio $\nu_{e}$ with fiber concentration $n L_{0}^{2}$ and fiber aspect ratio $\rho$. 


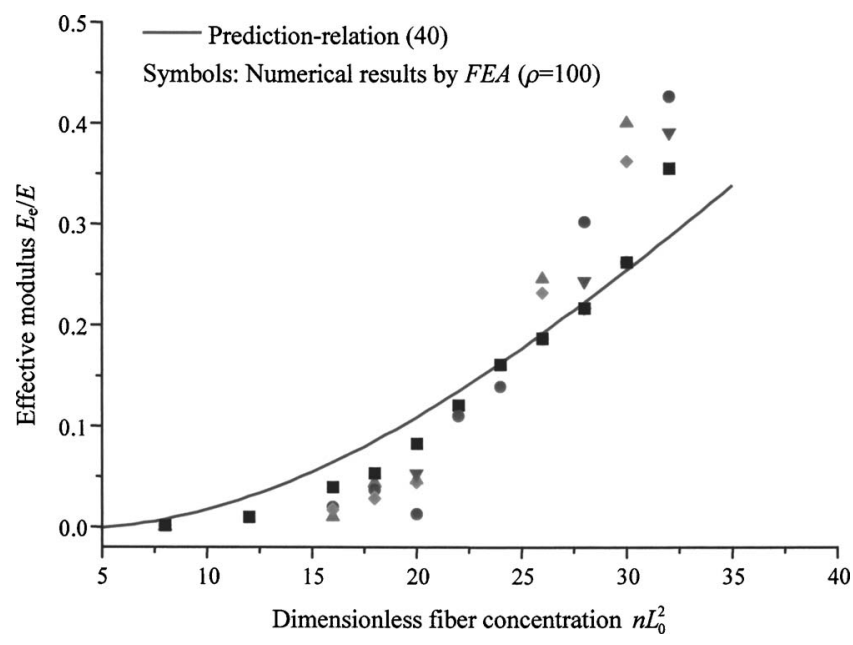

FIG. 9. Comparison of theoretical prediction of $E_{e} / E$ with finite element simulations.

tions, the beam elements in FEA are expected to overestimate the strain energy due to the very short length of the beam segments. The deformation of a typical FN calculated by FEA is shown in Fig. 10.

\section{SUMMARY}

A micromechanics model has been developed for the elastic stiffness of planar FNs. The model accounts for microscopic deformations of fiber segments of all possible lengths and orientations. Explicit expressions for the effective stiffness of random FNs have been derived based on the equivalency of the strain energy dissipated by the microscopic fiber deformations to the energy of the effective anisotropic continuum medium. The obtained constitutive relations can be used for the prediction of $\mathrm{FN}$ mechanical properties, scaling analysis, and optimization of FNs with

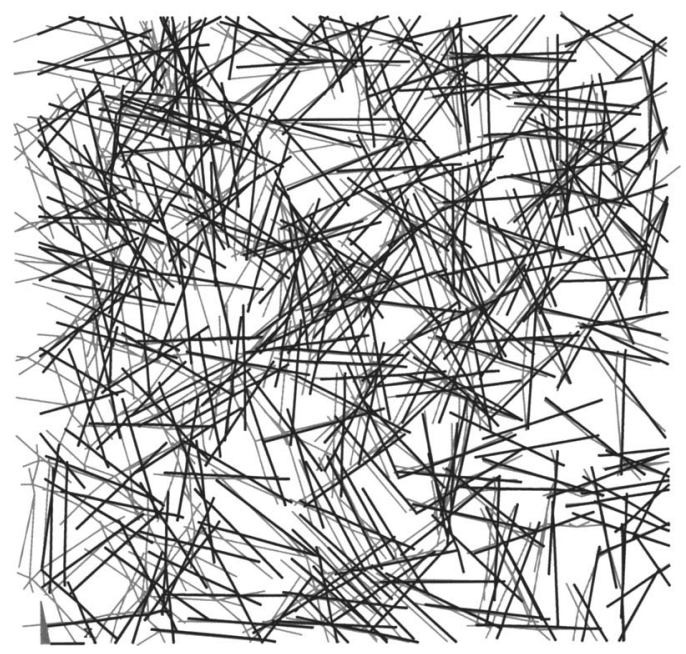

FIG. 10. Deformation of a typical fiber network under uniaxial tension $\left(n L_{0}^{2}=22\right)$ (fibers in dark color are those before deformation). strong contact bonds. The present scheme can be extended for the study of the effective stiffness of 3D fiber networks.

\section{ACKNOWLEDGMENTS}

Partial support of this work by the NSF, AFOSR, and ARO/ARL is gratefully acknowledged. Part of this study was presented at the 41st Annual Meeting of Society of Engineering Science (SES2004) in Lincoln, Nebraska, October 2004.

${ }^{1}$ C. W. Wang, L. Berhan, and A. M. Sastry, ASME J. Eng. Mater. Technol. 122, 450 (2000)

${ }^{2}$ C. W. Wang and A. M. Sastry, ASME J. Eng. Mater. Technol. 122, 460 (2000).

${ }^{3}$ J. Doshi and D. H. Reneker, J. Electrost. 35, 151 (1995).

${ }^{4}$ D. H. Reneker and I. Chun, Nanotechnology 7, 216 (1996).

${ }^{5}$ Y. Dzenis, Science 304, 1917 (2004).

${ }^{6}$ J. S. Kim and D. H. Reneker, Polym. Compos. 20, 124 (1999).

${ }^{7}$ Y. A. Dzenis and D. H. Reneker, U.S. Patent No. 626533 (2001).

${ }^{8}$ Y. A. Dzenis and Y. K. Wen, Mater. Res. Soc. Symp. Proc. 702, 173 (2002).

${ }^{9}$ Z. M. Huang, Y. Z. Zhang, M. Kotaki, and S. Ramakrishna, Compos. Sci. Technol. 63, 2223 (2003).

${ }^{10}$ C. T. J. Dodson, Br. J. Appl. Phys. 18, 1199 (1967).

${ }^{11}$ K. J. Niskanen and M. J. Alava, Phys. Rev. Lett. 73, 3475 (1994).

${ }^{12}$ E. K. O. Hellen, M. J. Alava, and K. J. Niskanen, J. Appl. Phys. 81, 6425 (1997).

${ }^{13}$ V. I. Raisanen, M. J. Alava, and R. M. Nieminen, J. Appl. Phys. 82, 3747 (1997).

${ }^{14}$ J. Astrom, M. Kellomaki, and J. Timonen, J. Phys. A 30, 6601 (1997).

${ }^{15}$ W. W. Sampson, J. Mater. Sci. 39, 2775 (2004).

${ }^{16}$ C. M. van Wyk, J. Text. Inst. 37, T285 (1946).

${ }^{17}$ T. Komori and K. Makishima, Text. Res. J. 47, 13 (1977).

${ }^{18}$ N. Pan and G. A. Carnaby, Text. Res. J. 59, 285 (1989).

${ }^{19}$ G. A. Carnaby and N. Pan, Text. Res. J. 59, 275 (1989).

${ }^{20}$ T. Komori and M. Itoh, Text. Res. J. 61, 420 (1991).

${ }^{21}$ T. Komori and M. Itoh, Text. Res. J. 61, 588 (1991).

${ }^{22}$ T. Komori, M. Itoh, and A. Takaku, Text. Res. J. 62, 567 (1992).

${ }^{23}$ D. H. Lee and G. A. Carnaby, Text. Res. J. 62, 185 (1992).

${ }^{24}$ N. Pan, J. Chen, M. Seo, and S. Backer, Text. Res. J. 67, 907 (1997).

${ }^{25}$ S. Toll and J.-A. E. Manson, ASME J. Appl. Mech. 62, 223 (1995).

${ }^{26}$ S. Toll, Polym. Eng. Sci. 38, 1337 (1998).

${ }^{27}$ H. L. Cox, Br. J. Appl. Phys. 3, 72 (1952).

${ }^{28}$ M. A. Narter, S. K. Batra, and D. R. Buchanan, Proc. R. Soc. London, Ser. A 455, 3543 (1999).

${ }^{29}$ J. Astrom, S. Saarinen, K. Niskanen, and J. Kurkijarvi, J. Appl. Phys. 75, 2383 (1994).

${ }^{30}$ J. A. Astrom, J. P. Makinen, M. J. Alava, and J. Timonen, Phys. Rev. E 61, 5550 (2000).

${ }^{31}$ J. A. Astrom, J. P. Makinen, H. Hirvonen, and J. Timonen, J. Appl. Phys. 88, 5056 (2000)

${ }^{32}$ A. M. Sastry, C. W. Wang, and L. Berhan, Key Eng. Mater. 200, 229 (2001).

${ }^{33}$ L. Berhan, Y. B. Yi, and A. M. Sastry, J. Appl. Phys. 95, 5027 (2004).

${ }^{34}$ L. Berhan, Y. B. Yi, A. M. Sastry, E. Munoz, M. Selvidge, and R. Baughman, J. Appl. Phys. 95, 4335 (2004).

${ }^{35}$ J. Astrom, S. Saarinen, and K. Niskanen, Europhys. Lett. 21, 557 (1993).

${ }^{36}$ V. I. Raisanen, M. J. Alava, K. J. Niskanen, and R. M. Nieminen, J. Mater. Res. 12, 2725 (1997).

${ }^{37}$ G. E. Pike and C. H. Seager, Phys. Rev. B 10, 1421 (1974).

${ }^{38}$ D. J. Jacobs and M. F. Thorpe, Phys. Rev. Lett. 75, 4051 (1995).

${ }^{39}$ C. Moukarzel and P. M. Duxbury, Phys. Rev. Lett. 75, 4055 (1995).

${ }^{40}$ S. Toll, S. Ranganathan, and S. G. Advani, J. Rheol. 37, 123 (1993).

${ }^{41}$ T. Mura, Micromehanics of Defects in Solids (Martinus Nijhoff, Boston, 1982).

${ }^{42}$ S. Nemat-Nasser and M. Hori, Micromechanics: Overall Properties of Heterogeneous Materials, 2nd ed. (Elsevier, Amsterdam, 1999).

${ }^{43}$ P. Boresi, R. J. Schmidt, and O. M. Sidebottom, Advanced Mechanics of Materials (Wiley, Toronto, 1992). 